\title{
Interference in above-threshold-ionization electron distributions from molecules
}

\author{
Jost Henkel, ${ }^{1,2}$ Manfred Lein, ${ }^{2}$ and Volker Engel ${ }^{1}$ \\ ${ }^{1}$ Institut für Physikalische und Theoretische Chemie, Universität Würzburg, and Röntgen Research Center for Complex Material Systems, Am \\ Hubland, D-97074 Würzburg, Germany \\ ${ }^{2}$ Institut für Theoretische Physik and Centre for Quantum Engineering and Space-Time Research (QUEST), Leibniz Universität Hannover
} Appelstraße 2, D-30167 Hannover, Germany

(Received 4 February 2011; published 19 May 2011)

\begin{abstract}
We present quantum-mechanical studies on above-threshold ionization of molecular ions in two and three dimensions. The momentum distributions show signatures of interfering emissions from the molecular centers. These structures deviate from a simple double-slit model that ignores the electron-ion interaction, but they are reproduced by an eikonal model. Such distortions of the interference pattern are partly responsible for the absence of clear interference patterns in the angle-integrated electron energy spectra.
\end{abstract}

DOI: 10.1103/PhysRevA.83.051401

PACS number(s): $33.20 . \mathrm{Xx}, 33.80 . \mathrm{Rv}, 42.50 . \mathrm{Hz}$

Above-threshold ionization (ATI) is one of the most fundamental phenomena occurring in the interaction of strong laser fields with atoms and molecules [1,2]. Briefly, the effect is that an electron (or several electrons $[3,4]$ ) absorbs more photons than needed to overcome the ionization threshold, leading to ATI peaks separated by the photon energy in the photoelectron kinetic-energy distribution. The ionization may proceed directly or indirectly via rescattering of the electron from the nuclei. For an extensive discussion of the theory of ATI, see the excellent review article by Becker et al. [5].

The first proposal toward molecular imaging based on ATI was made by Zuo, Bandrauk, and Corkum [6]. They determined photoelectron momentum distributions originating from multiphoton ionization of an $\mathrm{H}_{2}{ }^{+}$molecular ion with the nuclei fixed at different internuclear distances. Using a twodimensional model of $\mathrm{H}_{2}{ }^{+}$aligned parallel or perpendicular to the laser field, they found not only the characteristic ATI rings separated by the photon energy; additionally, they showed that under certain conditions regular patterns arise which are superimposed on the ATI rings. These features stem from the emission of electrons from different centers, leading to interferences in a similar way as they arise in a diffraction experiment.

In recent years, laser-induced diffraction in ATI has been discussed extensively [7-14]. These studies have mostly focused on the rescattered electrons for which the existence of diffraction patterns has been clearly demonstrated. The existence of interference in direct ionization is a separate question, which has been under debate recently. Although the calculated distributions by Zuo et al. [6] were explained in terms of direct interference, it must be noted that only a few ATI rings were investigated and the interference pattern was not very clear for the parallel configuration. Later, destructive twocenter interference was made responsible for the suppressed ionization of $\mathrm{O}_{2}$ molecules as compared to atoms with a similar ionization potential $[15,16]$. The interference effect has been confirmed in measurements of the molecular and atomic ATI spectra [17]. Measured angular distributions from $\mathrm{Ar}_{2}$ molecules differ significantly from distributions from Ar atoms [18]. However, the difference could not be fully explained by an interference model. Finally, the numerical results by Vanne and Saenz on $\mathrm{H}_{2}$ did not show the signature of two-center interference in the angle-integrated ATI spectrum [19]. This finding is not only in disagreement with the interference picture of [15-17], but raises also the question why the interferences found in the momentum distributions $[6,18]$ do not appear in the ATI spectrum.

In the present work, we use two-dimensional and threedimensional simulations for $\mathrm{H}_{2}{ }^{+}$to show that the electron momentum distributions exhibit clear interference patterns. They deviate from simple double-slit patterns, but they are reproduced by an eikonal model that takes the electron-ion interaction into account. The calculated energy spectra, on the other hand, do not show a clear interference pattern, confirming the previous result for $\mathrm{H}_{2}$ [19]. We show that the electron-ion interaction counteracts the visibility of interferences in the ATI spectra.

The importance of the electron-ion interaction has been recognized before and its effect has been successfully incorporated in the theory of laser-induced processes (see, e.g., the use of approximate Coulomb continuum functions in the ionization of molecules [20,21] or the eikonal model in strongfield ionization [22]). In the present work, we employ a simple version of the eikonal model to provide an easy interpretation of the two-dimensional (2D) and three-dimensional (3D) numerical momentum distributions.

We solve the time-dependent Schrödinger equation (TDSE) for one electron (atomic units are used)

$$
i \frac{\partial}{\partial t} \psi(\mathbf{r}, t)=\left[\hat{H}_{0}+\hat{W}(t)\right] \psi(\mathbf{r}, t)
$$

with the system Hamiltonian $\hat{H}_{0}=\frac{\hat{\mathbf{p}}^{2}}{2}+V_{N}(\mathbf{r})$, where $\hat{\mathbf{p}}$ denotes the electron momentum operator and $V_{N}$ is the potential created by $N$ fixed nuclei at positions $\mathbf{R}_{n}$ in the $(x, y)$ plane. We use the soft-core potential

$$
V_{N}(\mathbf{r})=-\sum_{n=1}^{N} \frac{1}{\sqrt{\left(1+\left|\mathbf{r}-\mathbf{R}_{\mathbf{n}}\right|^{2}\right)}} .
$$

The dipole interaction with a linearly polarized laser field is included in the velocity gauge as

$$
\hat{W}(t)=\hat{\mathbf{p}} \mathbf{A}(t)=-\hat{\mathbf{p}} \boldsymbol{\epsilon} \int_{0}^{t} d t^{\prime} E_{0} f\left(t^{\prime}\right) \sin \left(\omega t^{\prime}\right),
$$


with polarization vector $\boldsymbol{\epsilon}$, field strength $E_{0}$, frequency $\omega$, and pulse envelope $f(t)$. We employ a laser wavelength of $532 \mathrm{~nm}$ (photon energy $2.33 \mathrm{eV}$ ) and a field amplitude of $E_{0}=$ 0.06 a.u., corresponding to an intensity of $0.126 \mathrm{PW} / \mathrm{cm}^{2}$. The field envelope is turned on and off linearly over two optical cycles $T=2 \pi / \omega$ and is kept constant at $f(t)=1$ over 12 cycles. The simulation is run for five additional cycles without field to collect slow photoelectrons. The TDSE is solved numerically using the split-operator technique [23] in 2D and 3D. Initially, the system is in its ground state which is calculated via imaginary-time propagation [24]. The 2D numerical grid size is 1600 a.u. along each axis with a spacing of 0.39 a.u. (4096 grid points). The 3D grid gize is 800 a.u. with a spacing of 1.6 a.u. (512 grid points). Despite the coarse 3D spacing, the comparison of the results with the numerically accurate $2 \mathrm{D}$ results shows agreement, indicating that the results are convergent with respect to the numerical parameters. The laser field is aligned along the $y$ axis of the coordinate system. We divide the grid into an interaction (IA) and an asymptotic (AS) region. In the AS region the electron is treated as a free particle interacting with the external laser field [25,26]. The 2D wave function is decomposed into an inner and outer part by multiplication with a window function $w_{2 \mathrm{D}}(\mathbf{r})=$ $w(x) w(y)$, where $w(x)=\exp \left[-\beta\left(|x|-r_{c}\right)^{2} \theta\left(|x|-r_{c}\right)\right]$, with $r_{c}=200$ a.u., $\beta=10^{-4}$ a.u., and the Heaviside function $\theta$. Similarly, in the 3D case, we use $w_{3 \mathrm{D}}(\mathbf{r})=w(x) w(y) w(z)$. The interaction part $\psi_{\mathrm{IA}}(\mathbf{r}, t)$ of the wave function is propagated with the complete Hamiltonian and the time evolution of the asymptotic part is carried out in momentum space with the Hamiltonian of the free electron in the laser field. The windowing is performed at each timestep $t_{n}$ and the obtained new fractions of the asymptotic wave function are added coherently to the momentum-space function $\tilde{\psi}_{\mathrm{AS}}\left(\mathbf{p}, t_{n}\right)$.

The photoelectron momentum distribution is calculated at the final time $t_{\max }=21 T \approx 37 \mathrm{fs}$ as

$$
\sigma_{\mathrm{AS}}(\mathbf{p})=\left|\tilde{\psi}_{\mathrm{AS}}\left(\mathbf{p}, t_{\max }\right)\right|^{2}
$$

whereas the time-dependent momentum distribution for electrons still located in the interaction region is

$$
\sigma_{\mathrm{IA}}(\mathbf{v}, t)=\left|\tilde{\psi}_{\mathrm{IA}}(\mathbf{v}-\mathbf{A}(t), t)\right|^{2},
$$

with the kinetic momentum $\mathbf{v}=\mathbf{p}+\mathbf{A}(t)$ and the momentumspace wave function $\tilde{\psi}_{\text {IA }}$.

For better interpretation of the numerical results, we consider two models for describing the interference between emissions from different centers. Ignoring the effect of the laser field on the electron motion, we calculate the phases of the electron paths starting with the same velocity from the $N$ centers. The coherent summation of these $N$ contributions leads to interference minima and maxima depending on the relative phases. The paths are parallel straight lines starting at the centers with an electron velocity corresponding to the desired final electron momentum and leading toward infinity. In the double-slit model, the electron-ion interaction is neglected. For a diatomic system with internuclear vector $\mathbf{R}$, this leads to the usual double-slit interference

$$
\sigma_{\mathrm{AS}} \sim \cos ^{2}(\mathbf{p R} / 2)
$$

Second, we consider an eikonal model taking the electron-ion interaction into account [27]. While the electron is still assumed to move on a straight line, the instantaneous velocity $u$ depends on the position as $u(\mathbf{r})=\sqrt{2\left[E-V_{N}(\mathbf{r})\right]}$ due to energy conservation with $E=\mathbf{p}^{2} / 2$ being the total energy for the final momentum $\mathbf{p}$. The phase $\varphi_{n}$ acquired along the path starting from the nucleus at $\mathbf{R}_{n}$ is calculated as an action integral along the path, that is, $\varphi_{n}=\int_{\mathbf{R}_{n}}^{\mathbf{r}_{n}} u(\mathbf{r}) d s$, with endpoints $\mathbf{r}_{n}$ far away from the nuclei. The endpoints of the different paths must lie on the same plane-wave front, which is perpendicular to the velocity. The momentum $\mathbf{p}$ is scanned with a spacing of $1.6 \times 10^{-3}$ a.u. in each direction. A coherent summation over the different paths yields

$$
\sigma_{\mathrm{AS}} \sim\left|\sum_{n} e^{i \varphi_{n}}\right|^{2} .
$$

In a heteronuclear or polyatomic molecule, the initial wave function may be distributed unequally over different centers. In this case, each contribution is weighted by the value of the wave function at the corresponding core

$$
\sigma_{\mathrm{AS}} \sim\left|\sum_{n} \psi_{\mathrm{IA}}\left(\mathbf{R}_{n}, 0\right) e^{i \varphi_{n}}\right|^{2} .
$$

In Fig. 1, we show momentum distributions obtained from a 2D TDSE calculation for $\mathrm{H}_{2}{ }^{+}$at an internuclear distance of $R=20$ a.u. We first discuss the dynamics of the momentum-space wave function in the interaction region, Figs. 1(a) through (e). At all times a clear interference pattern is found, which has approximately the form of nodal lines along the $p_{y}$ direction. This structure appears clearly already in the initial ground state at $t=0$. The reason is that the ground state $\psi_{0}$ is well described by a linear combination of atomic orbitals $\phi$

$$
\psi_{0}(\mathbf{r}) \sim \phi(\mathbf{r}+\mathbf{R} / 2)+\phi(\mathbf{r}-\mathbf{R} / 2),
$$

with the momentum-space wave function $\tilde{\psi}_{0}(\mathbf{p}) \sim \tilde{\phi}(\mathbf{p})$ $\cos (\mathbf{p R} / 2)$. This yields the same interference pattern as

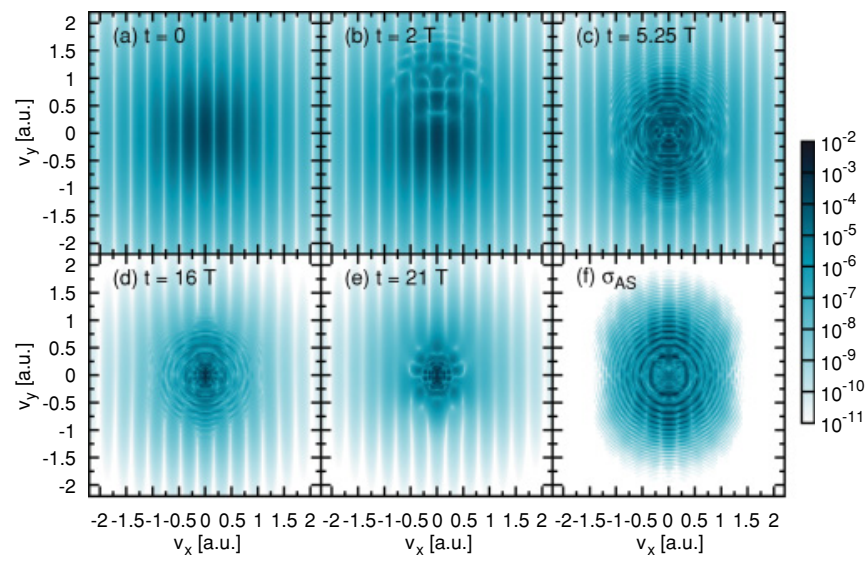

FIG. 1. (Color online) Momentum distributions for $\mathrm{H}_{2}{ }^{+}$at the internuclear distances $R=20$ a.u. aligned perpendicular to the laser field (logarithmic color scale). (a-e) Time-dependent distributions in the interaction region at different times $t$, as indicated. The laser field is turned off at $t=16 T$. (f) Asymptotic photoelectron distribution. 


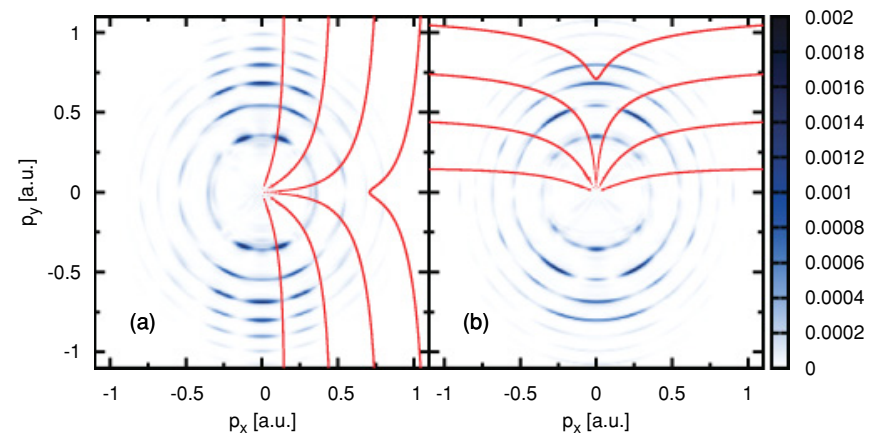

FIG. 2. (Color online) Asymptotic momentum distributions $\sigma_{\mathrm{AS}}$ for $\mathrm{H}_{2}{ }^{+}$at $R=20$ a.u. with (a) perpendicular alignment and (b) parallel alignment of the molecular axis to the laser field. The red lines indicate the minima predicted by the eikonal model, Eq. (7).

expected from the double-slit model for the ionizing parts of the wave function. For the arrangement of Fig. 1 with two nuclei located at $\mathbf{R}_{1}=\left(\frac{R}{2}, 0\right)$ and $\mathbf{R}_{2}=\left(-\frac{R}{2}, 0\right)$, destructive interference is predicted at $p_{x}=(2 n+1) \pi / R$ with $n=$ $0,1, \ldots$ For $R=20$ a.u. this yields values of $p_{x}=0.157$ a.u., 0.471 a.u. for $n=0,1$ in perfect agreement with the numerical results in Fig. 1. A closer inspection of Fig. 1 shows that the ground state is superimposed by the wavepackets corresponding to ionization. The latter follow the oscillations of the vector potential along the polarization axis and show the expected ATI rings defined by $p_{y}^{2} / 2+p_{x}^{2} / 2=E$ with total final energy $E$. While the initial state shows straight nodal lines, the ionizing wavepackets exhibit a bent diffraction pattern, see Fig. 1(c). This becomes more apparent in the asymptotic distribution, Fig. 1(f), which contains no traces of the bound states and only displays ATI rings modulated with interference patterns. Clearly, the simple double-slit formula Eq. (6) cannot reproduce these features correctly. Using the eikonal model we are able to describe the pattern more accurately. In Fig. 2, we show results for both perpendicular and parallel alignments of $\mathrm{H}_{2}{ }^{+}$relative to the field. The red lines show the zeros of the surface obtained from Eq. (7). These lines reproduce the minima in the ATI rings save a few exceptions such as the innermost ring in perpendicular alignment. It is remarkable that the numerical interference pattern is reproduced by a model that does not depend on the laser field. The agreement may degrade for higher intensity or longer wavelength.

Next, we turn to the case of a linear arrangement of three nuclei with a distance of $R=10$ a.u. between adjacent nuclei. The momentum distributions are displayed in Fig. 3. In the eikonal model, we use the weighted sum according to Eq. (8). In contrast to the two-center case, the interference is never fully destructive. Nevertheless, the contour lines in Fig. 3 clearly show trenches of suppressed yield. Similar to the two-center case, these trenches lie along curves that are bent inward near the origin, but for high values of $p$ they approach the straight lines predicted by Eq. (6). The numerical interference pattern agrees well with the model.

Vanne and Saenz have recently investigated the question whether interference minima appear in the angle-integrated ATI spectra [19]. Under the assumption that the electrons are predominantly ejected along the laser polarization axis,

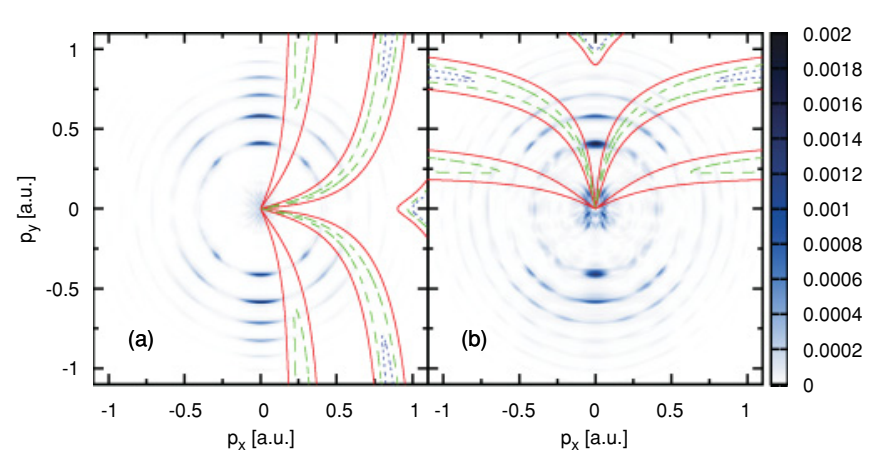

FIG. 3. (Color online) Asymptotic momentum distributions $\sigma_{\mathrm{AS}}$ for $\mathrm{H}_{3}{ }^{2+}$. (a) Perpendicular alignment with the nuclei positioned at $R_{1}=(-10,0), R_{2}=(0,0)$, and $R_{3}=(10,0)$. (b) Parallel alignment with $R_{1}=(0,-10), R_{2}=(0,0)$, and $R_{3}=(0,10)$. The red straight, green dashed, and blue dotted lines are contour lines at the values 0.96 , 0.93 , and 0.92 , respectively, of the surface given by the three-center eikonal model, Eq. (8), which yields values between 0.9 and 1.1 .

one expects that the interference minima discussed above persist after integration over the angle. In the double-slit model for $\mathrm{H}_{2}{ }^{+}$, this leads to minima at $E_{n}=\pi^{2}(2 n+1)^{2} /\left(2 R^{2}\right)$ in the ratio between the spectra for parallel and perpendicular alignments. This behavior is also predicted by the molecular strong-field approximation in the velocity gauge [19]. However, the TDSE results for $\mathrm{H}_{2}$ at $R=3$ a.u. did not confirm this interference pattern [19]. In Fig. 4 we show the ATI spectra for parallel and perpendicular alignments of $2 \mathrm{D} \mathrm{H}_{2}{ }^{+}$as well as the ratio between the two spectra. Although some modulations of the ratio are seen in the low-energy region, there is no resemblance with the interference pattern predicted by the double-slit model. From Fig. 2, we understand that there are two reasons why the interference pattern in the momentum distribution does not survive the integration over angle. First, the momentum distributions are not focused enough along the polarization axis to allow a unique correspondence between electron energy and momentum component along the molecular axis. Second, the nodal lines of the interference patterns are distorted by the electron-ion interaction. The curvature is such that it tends to wash out the angle-integrated interference pattern.

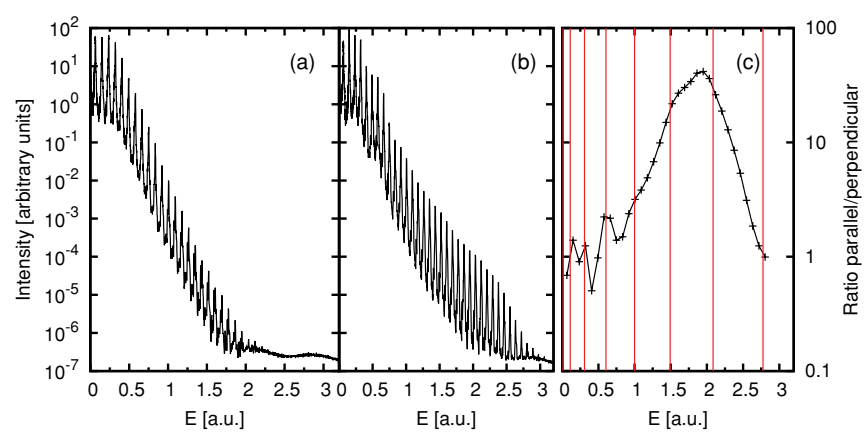

FIG. 4. (Color online) Energy spectra obtained from Fig. 2 by integration over emission angle. (a) Spectrum for perpendicular alignment. (b) Spectrum for parallel alignment. (c) Ratio of parallel to perpendicular spectrums. The red lines are the positions of the minima predicted by $E_{n}=\pi^{2}(2 n+1)^{2} /\left(2 R^{2}\right)$. 


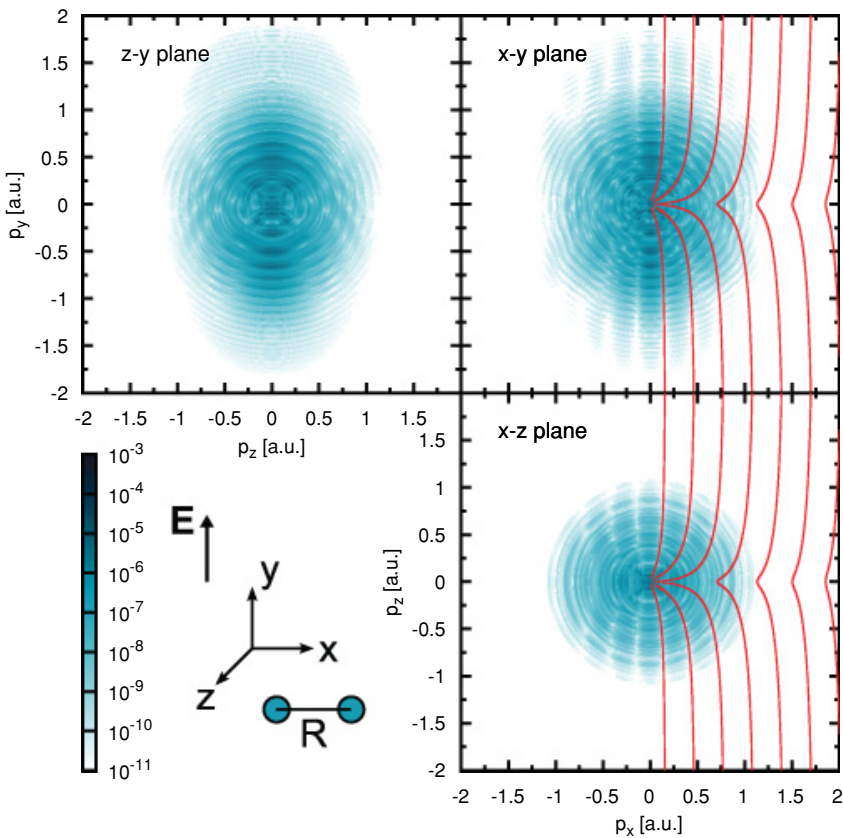

FIG. 5. (Color online) Asymptotic momentum distributions $\sigma_{\mathrm{AS}}$ for $\mathrm{H}_{2}{ }^{+}$perpendicular to the laser field as in Fig. 2, obtained as 2D cuts from the $3 \mathrm{D}$ calculation (logarithmic color scale). Also shown is the molecule-field geometry.

We finally turn to a $3 \mathrm{D}$ calculation to investigate if the interference patterns persist if the full dimensionality is taken into account. Figure 5 shows 2D cuts through the 3D momentum distributions. The molecular axis is along the $x$ axis [i.e., perpendicular to the laser field ( $y$ axis)], as is illustrated in the figure. Interference patterns are visible in the $x-y$ and $x-z$ planes The predictions made by the eikonal model (red lines in Fig. 5) are the same as in Fig. 2 and give an adequate description of the interference pattern. The slight deviations for momenta with $p_{x}>0.5$ a.u. may be related to the low spatial resolution used in the $3 \mathrm{D}$ calculation. The results show that, for the present arrangement, the $2 \mathrm{D}$ treatment is a good approximation to the complete 3D calculation when studying only the dynamics in the $x-y$ plane.

To summarize, the TDSE for 2D and 3D molecular ions at large internuclear distances has been solved numerically to investigate interference in multiphoton ionization. The ATI rings are modulated with interference patterns due to the two- or multicenter character of the molecular ions. The development of the momentum distributions in time shows that the initial state has an interference pattern of straight lines whereas the photoelectron momentum distributions exhibit a distorted pattern. Using an eikonal model that takes the electron-ion interaction into account, we can reproduce these structures in both 2D and 3D calculations. The distortion is such that the interference pattern is washed out when the momentum distribution is integrated over angle. This is partly responsible for the absence of a clear interference pattern in the ATI spectrum.

J.H. acknowledges financial support from the Studienstiftung des Deutschen Volkes. This work was supported by the Deutsche Forschungsgemeinschaft. Funding from the EU Marie Curie Initial Training Network FASTQUAST is acknowledged.
[1] P. Agostini, F. Fabre, G. Mainfray, G. Petite, and N. K. Rahman, Phys. Rev. Lett. 42, 1127 (1979).

[2] J. H. Eberly, J. Javanainen, and K. Rzążewski, Phys. Rep. 204, 331 (1991).

[3] J. S. Parker, L. R. Moore, K. J. Meharg, D. Dundas, and K. T. Taylor, J. Phys. B 34, L69 (2001).

[4] M. Lein, E. K. U. Gross, and V. Engel, Phys. Rev. A 64, 023406 (2001).

[5] W. Becker, F. Grasbon, R. Kopold, D. B. Milošević, G. G. Paulus, and H. Walther, Adv. At. Mol. Opt. Phys. 48, 35 (2002).

[6] T. Zuo, A. D. Bandrauk, and P. B. Corkum, Chem. Phys. Lett. 259, 313 (1996).

[7] M. Lein, J. P. Marangos, and P. L. Knight, Phys. Rev. A 66, 051404(R) (2002).

[8] S. N. Yurchenko, S. Patchkovskii, I. V. Litvinyuk, P. B. Corkum, and G. L. Yudin, Phys. Rev. Lett. 93, 223003 (2004).

[9] M. Spanner, O. Smirnova, P. B. Corkum, and M. Y. Ivanov, J. Phys. B 37, L243 (2004).

[10] H. Hetzheim, C. Figueira de Morisson Faria, W. Becker, Phys. Rev. A 76, 023418 (2007).

[11] M. Meckel et al., Science 320, 1478 (2008).

[12] M. Okunishi et al., Phys. Rev. Lett. 103, 043001 (2009).
[13] C. D. Lin, A.-T. Le, Z. Chen, T. Morishita, and R. Lucchese, J. Phys. B 43, 122001 (2010).

[14] F. Krasniqi, B. Najjari, L. Strüder, D. Rolles, A. Voitkiv, and J. Ullrich, Phys. Rev. A 81, 033411 (2010).

[15] J. Muth-Böhm, A. Becker, and F. H. M. Faisal, Phys. Rev. Lett. 85, 2280 (2000).

[16] X. Ren, J. Zhang, P. Liu, Y. Wang, and Z. Xu, Phys. Rev. A 78, 043411 (2008).

[17] F. Grasbon, G. G. Paulus, S. L. Chin, H. Walther, J. Muth-Böhm, A. Becker, and F. H. M. Faisal, Phys. Rev. A 63, 041402 (2001).

[18] Z. Ansari et al., New J. Phys. 10, 093027 (2008).

[19] Y. V. Vanne and A. Saenz, Phys. Rev. A 82, 011403(R) (2010).

[20] G. L. Yudin, S. Chelkowski, and A. D. Bandrauk, J. Phys. B 39, L17 (2006).

[21] M. F. Ciappina and W. R. Cravero, J. Mod. Opt. 56, 11 (2009).

[22] O. Smirnova, M. Spanner, and M. Y. Ivanov, Phys. Rev. A 77, 033407 (2008).

[23] M. D. Feit, J. A. Fleck, and A. Steiger, J. Comput. Phys. 47, 412 (1982).

[24] R. Kosloff and H. Tal-Ezer, Chem. Phys. Lett. 127, 223 (1986).

[25] R. Heather and H. Metiu, J. Chem. Phys. 86, 5009 (1987).

[26] R. Heather, Comput. Phys. Commun. 63, 446 (1991).

[27] L. I. Schiff, Quantum Mechanics (McGraw-Hill, Tokyo, 1968). 